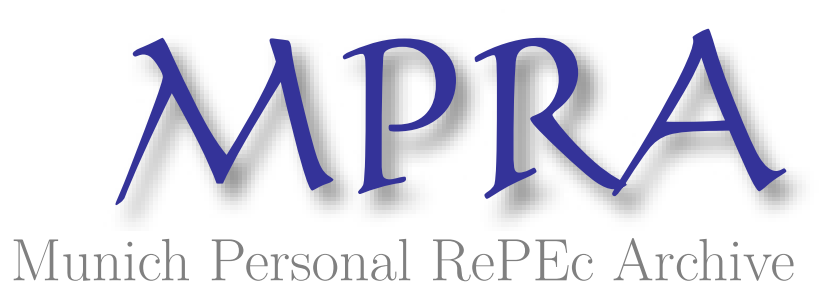

\title{
Two measures of organizational flexibility
}

Fioretti, Guido

University of Bologna

9 April 2008

Online at https://mpra.ub.uni-muenchen.de/16961/

MPRA Paper No. 16961, posted 28 Aug 2009 20:36 UTC 


\title{
Two Measures of Organizational Flexibility
}

\author{
Guido Fioretti \\ University of Bologna \\ Department of Management Science \\ guido.fioretti@unibo.it
}

December 17, 2008

\begin{abstract}
Since the second half of the past century, increasingly flexible organizational forms are appearing among firms. However, while hierarchies are easily described, too few mathematical tools are available for flexible organizations.

In this article, two measures are proposed in order to assess the state and trend of flexible organizations. The first of these measures is based on information waste, which occurs whenever information is classified into categories. The second measure is based on duplication of operations.

The underlying idea is that firms have an endogenous drive towards organizational configurations where waste of information and duplication of operations are minimized. However, environmental uncertainty may require some flexibility, which is ensured by cognitive processes that discard some information as well as by parallel undertaking of similar actions.

Both cognitive processes and parallel action are firm-specific features that only make sense within the knowledge-based view of the firm. However, in the special case where information waste and duplication of operations reach zero a firm can be described as an information processor, as contractualist views prescribe.
\end{abstract}

JEL: D23, L20

Keywords: Flexible organizations, New organizational forms, Theory of the Firm

\section{Introduction}

Since several decades, many firms are shifting towards structures that are ever more flexible, decentralized, reticular or otherwise defined but, in some sense, different from classical hierarchies [20]. Possibly, even the classical shift from functional to multidivisional structures may be interpreted as a part of this secular trend [5]. Wherever its origin may be dated back, this process clearly accelerated since the 1980s, giving rise to a series of buzzwords such as "kanban system", "teamwork", "production islands", "flat structures", "network firm" and others more or less directly related to the idea of increasing flexibility.

Henceforth, the expression "flexible organization" will be used to capture organizational forms that, under some respect, depart from bureaucracies. This article takes 
the stand that, beyond the many managerial fads and myths surrounding flexibility and flexible organizations, a real trend towards flexibility does exist.

According to many observers, a trend of this kind is mainly due to increasing environmental unpredictability induced by increasing variety of tastes and products [14]. Indeed, firms operating in predictable environments escaped the general trend - a good example being that of large companies operating in the mining sector that never switched to the multidivisional structure [9].

In any case, increasing flexibility should not be seen as a compelling trend for all firms. It is clear that, although some firms are developing highly decentralized and flexible forms, others just softened the bureaucratic procedures that they had adopted in the 1960s and 1970s, while still others are by no means affected by the rush towards flexibility. One may conclude that albeit a trend does exist, this does not imply a radical transformation of all firms but rather the emergence of a novel organizational form that will possibly coexist with the previous ones.

This novel organizational form rejects a classical prescription of organization theory, namely, that the productive core of a firm should be isolated from all unpredictable environmental variability [36]. Rather than buffering disturbances by means of inventories and standardized procedures, these firms accept that unpredictable signals enter their daily operations in order to exploit market niches — for instance, a firm may care for customizations and dedicated production lots in order to serve particular needs.

It is obvious that a firm that sets out to exploit an unpredictable environment must entail a wider array of behavioral patterns than a firm designed to cope with a stable and predictable environment. Furthermore, Ashby's principle of requisite variety may suggest that the array of behavioral patterns of such a firm should be even larger than that of the environment itself [3].

These quite general considerations suggest that firms characterized by a flexible organization should exhibit both a high cognitive ability, in order to understand novel situations, and a substantial innovative ability, in order to cope with novel situations. These two features may be expressed as follows:

1. A flexible firm must be able to understand and adapt to novel situations. Therefore, it must be able to classify a huge amount of information into categories, adapt its categories to the changing environment and develop proper patterns of action. Since classification implies that slightly different pieces of information are considered as equivalent to one another, information classification implies that some information is wasted in the process.

2. A flexible firm must be able to explore novel arrangements of its assets in order to satisfy novel needs. Thus, it must be able to try novel combinations and sequencings of its operations. Since by doing so it may occur that different parts of the firm explore the same combination and sequencing of operations, some unnecessary duplication of operations may take place.

The above issues regard (1) a firm's ability to recognize the relevant features of an unpredictable environment, and (2) its ability to explore appropriate reactions. Thus, they constitute sensible and valuable characteristics. 
However, both of them have drawbacks. In fact, with respect to a firm operating in a predictable environment it appears that a firm capable of recognizing novelties and developing appropriate responses may (1) waste too much information, and (2) duplicate too many operations.

Thus, under these respects the firms operating in unpredictable environments are less efficient than firms operating in predictable environments. Provided that firms seek efficiency, it follows that firms have a tendency to reduce information waste and duplication of operations, though the unpredictability of their environment may prevent them from reaching a state where no information is wasted and no operation is duplicated.

Thus, information waste and duplication of operations may play for the study of organizational forms a similar role as utility and profit functions in economics [8], fitness functions in evolutionary theory, gravitational and electrical potential functions in physics. All of them are Lyapunov functions (see Appendix A) describing the tendency of a system towards a stable equilibrium state.

Lyapunov functions describe a tendency that is not necessarily followed, but that nevertheless exerts a pressure pointing to a stable equilibrium. So microeconomics depicts firms as profit maximizers, evolutionary theory depicts species as fitness maximizers and physics describes falling bodies as potential minimizers; however, firms may not reach maximum profit because of management inefficiencies, exogenous constraints or other reasons, species may not reach maximum fitness because some evolutionary jumps may be unlikely or impossible, and some falling bodies may be impaired from reaching the soil by forces acting in the opposite direction. The existence of a Lyapunov function does not imply that the stable equilibrium will necessarily be reached before the system undergoes a transformation that changes the equilibrium itself. It just ensures that a tendency towards that equilibrium exists.

The current trend towards flexible organizations is generally interpreted as due to the fact that the environments where firms operate become ever less predictable with respect to managerial capabilities. Coherence with this interpretation implies that the natural tendency acts in the opposite direction, i.e. towards rigid, perfectly planned organizations where no information is wasted and no operations are duplicated. If the opposite trend is observed, this can only be due to increasingly unpredictable environments coupled with the bounded rationality of decision-makers. Thus, we set out to characterize flexibility by means of Lyapunov functions that express natural tendencies that operate when the environment is predictable, but that can be offset if the environment becomes unpredictable.

Consider the classical case of Toyota, which in the 1980s was able to develop new models faster than any American or Japanese competitor. Allegedly [38], this happened because within this firm (i) technical specifications fluctuated within broad ranges that were narrowed as late as possible (broad categories that waste a large amount of information), and (ii) a large number of alternative projects was carried out at the same time (duplication of operations).

However, the success of Toyota cannot be ascribed to greater information waste and greater duplication of operations in themselves, but to greater environmental unpredictability making information waste and duplication of operations costly but valuable features. This implicitly means that its U.S. competitors had actually achieved a 
higher degree of structural optimality with respect to an environment that was supposed to be stable but, alas, started to change in unpredictable ways. In a more predictable environment, such as those prevailing in the 1960s, flexibility was useless. According to the proposed interpretation the fact that at Toyota technical specifications fluctuated and several alternative projects were carried out at the same time is not good in itself, but had the advantage of enabling greater flexibility.

The idea underlying this article is that, since wasting information and duplicating operations are not good things by themselves, a tendency exists in all organizations to minimize them. However, the stable equilibrium where no information is wasted and no operation is duplicated can only be reached if the environment is perfectly predictable, for only if its environment is perfectly predictable a firm can afford not to be flexible at all. In general, the less predictable the environment, the more information is wasted, the more operations are duplicated, and the more flexible the organization.

If these two Lyapunov functions provide a valuable synthetic description of the distance of flexible organizational forms from the ideal organization of a firm operating in a predictable environment, then they can be used to compare alternative organizational forms and to evaluate their performance across environments. Similarly to comparisons of levels of utility and levels of profits, they may enable a comparative statics of organizational forms.

The ensuing section 2 defines and expounds the two proposed measures of flexibility. Section 3 sketches their possible application to numerical and empirical examples. Finally, section 4 frames this formalism with respect to the theories of the firm.

\section{Two Lyapunov Functions}

Let us conceive a firm as composed by organizational units that may entail both men and machines. Let us assume that each organizational unit is endowed with (1) a set of categories to classify information, (2) a set of pieces of information to be produced, and (3) a rule that specifies on what occasions a particular piece of information should be produced.

The categories represent the situations that decision-makers endowed with particular machines are able to recognize. For instance, a press operator may recognize any metal sheet as pertaining to him, whereas he would refuse paperwork as pertaining to somebody else.

The rule specifies which operation an organizational unit should carry out when certain situations are recognized. For instance our worker at the press, after recognizing a metal sheet may put it under the press, activate it and obtain an object with the desired shape.

The output of an organizational unit may be a physical object but also a piece of software or a communication. In any case, it is perceived by the receiving unit as a piece of information. For instance, for the press operator in the above example the metal sheet entails the information "this sheet must be shaped".

The above description of organizational units is quite general. The operations carried out by an organizational unit consist of producing as output a piece of information when a piece of information is received as input. The input information is classified by 


$\left[\begin{array}{l}\# \\ 1 \\ \# \\ 0\end{array}\right]$

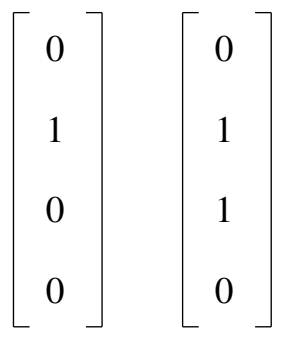

$\left[\begin{array}{l}1 \\ 1 \\ 0 \\ 0\end{array}\right]$

$\left[\begin{array}{l}1 \\ 1 \\ 1 \\ 0\end{array}\right]$

Figure 1: A category with two "don't care" characters and the four information strings that it classifies.

a category and the decision concerning which information should be issued as output is made by means of a rule.

Taking inspiration from classifier systems [11] [12], let us represent both categories and information by means of strings of characters that can be zeros, ones, or "don't care" characters \#. ${ }^{1}$ Henceforth we shall also employ the terms "category string" and "information string", respectively.

Category strings must entail at least one \#-character. In fact, categories are strings that match all information strings that have zeros and ones in the same positions where they have zeros and ones, while it does not matter which character information strings have where category strings have a \#. For instance, the category string depicted in the left half of figure 1 is able to classify the four information strings depicted in the right half of the picture. One may also visualize a category string as a container that collects all information strings that have zeros and ones in its same positions.

Information strings may have \#-characters as well. In this case, \#-characters represent the unpredictability of the corresponding information bit. Thus, if an information string with a \# is issued, this can only be classified by a category string that has a \# in the same position.

Let $H$ denote the number of different category strings owned by all organizational units of the firm. Let $K$ denote the number of different information strings that can be produced by all organizational units of the firm. Since categories are there in order to classify information, it must be $H<K$.

Organizational units may be so simple to have just one category string and be able to produce just one kind of category string. This may be e.g. the case of a specialized machine, a worker on the conveyor belt, or a department in a strongly bureaucratic organization.

In general, an organizational unit may own several categories, each of a different kind, that enable it to process different information strings. This may be the case of a machine that can be endowed with different tools, a worker with multiple skills, or a problem-solving unit where different specialties are represented.

Connections between any two organizational units take place with varying frequency depending on hierarchical relations, time required to carry out operations, relat-

\footnotetext{
${ }^{1}$ Classifier systems employ the terms "condition" and "action" where we said "category" and "information", respectively.
} 
edness of activities, patterns of acquaintance or else. If we assume that the connections between any two organizational units only depend on what category strings they own and what information strings they produce, then the structure of relationships within the firm can be expressed by the probabilities that a category string of type $h$ classifies an information string of type $k, \forall h=1,2, \ldots H$ and $\forall k=1,2, \ldots K$. Henceforth, let $p_{h k}$ denote the probability that a category string of type $h$ classifies an information string of type $k$.

The existence of a Lyapunov function implies that the operations carried out by the units and the probabilities $p_{h k}$ have a tendency towards an equilibrium configuration. If the Lyapunov function rests on structural properties, this equilibrium state is structurally optimal.

A firm can attain structural optimality if it finds itself in a predictable environment and if its decision-makers are capable of making predictions. Henceforth, these concepts will be used with the following meaning:

Predictability The environment of a firm is predictable if it behaves according to laws that are known by the firm, and if these laws do not require unavailable data in order to be applied.

Rationality Within a firm, decision-makers are rational if they make use of all available information to attain their goals.

Clearly, rational decision-makers and predictable environments are an abstraction. These abstractions are made in order to claim that, if the environment is predictable and the decision-makers are rational, then a firm tends to a stable equilibrium state where no information is wasted and no operations are duplicated.

In the following two subsections, two Lyapunov functions will be introduced, that describe the natural tendency of an organization to a state where no information is wasted and no operation is duplicated. For the idea of defining Lyapunov functions to describe the evolution of a decentralized economic system, Allais [1] has to be credited.

\subsection{Information Waste}

Let specificity denote the number of non-\# characters in a string. Let $s_{h}$ denote the specificity of category string $h$, where $h=1,2, \ldots H$, and let $z_{k}$ denote the specificity of information string $k$, where $k=1,2, \ldots K$.

Let us define the information waste when the category string $h$ classifies the information string $k$ as follows:

$$
u_{h k}=z_{k}-s_{h}
$$

Note that since $z_{k} \geq s_{h}$, it follows that $u_{h k} \geq 0$.

The waste of information in the whole firm is the sum of the information wasted at all connections. However, since connections occur with specific probabilities, the addends of this sum must be weighted:

$$
U=\sum_{h k} p_{h k} u_{h k}
$$


If a firm starts with a structure $\mathcal{S}_{*}$ and arrives at a structure $\mathcal{S}^{*}$, the corresponding variation of information waste is $\Delta U$ :

$$
\Delta U=\sum_{S_{i}=S_{*}}^{S^{*}} \Delta U_{i}
$$

where $\Delta U_{i}=\left.U\right|_{\mathcal{S}_{i}}-\left.U\right|_{\mathcal{S}_{i-1}}$ and where the series of structures $\left\{S_{i}\right\}$ extends from $\mathcal{S}_{*}$ to $S^{*}$.

If $\Delta U_{i}<0$ for $\forall i$, then the organization has a spontaneous tendency to move from structure $\mathcal{S}_{*}$ to structure $\mathcal{S}^{*}$.

\subsection{Operations Duplication}

The operations carried out by an organizational unit are the procedures it follows in order to produce an information string out of an input classified by one of its category strings. However, we do not observe the functioning of organizational units, but only the categories that they employ and the information that they produce. Thus, duplication of operations should be measured in these terms.

In order to consider the operations carried out by an organizational unit in terms of the categories that it employs and the information that it produces, we must consider chains of at least three units. Among two chains of three units, we can observe whether their central units duplicate their operations in the sense that they employ the same category string and produce the same information string.

Let us consider operations duplication by organizational units that classify information by means of category strings of type $h_{1}$ and produce information strings of type $k_{1}$. Let us consider chains of three organizational units that include the above units in the middle. Let the stream of information be $k \rightarrow h_{1} \mapsto k_{1} \rightarrow h$, where $k$ is the information string produced by the first unit and $h$ is the category string employed by the third unit, the symbol " $\rightarrow$ " denotes a connection between two organizational units whilst “ $\mapsto$ " denotes the operations carried out by the central unit in the chain.

Duplication of the operations carried out by one organizational unit takes place if two chains of three organizational units are linked by identical category strings and identical information strings. The probability that two chains of three units duplicate the operations of their central unit is:

$$
v_{1}^{2}=\frac{1}{H K} \sum_{h, h_{1}=1}^{H} \sum_{k, k_{1}=1}^{K}\left(p_{h k_{1}} p_{h_{1} k}\right)^{2}
$$

where coefficient $1 / H K$ ensures that $0 \leq v_{1}^{2} \leq 1$.

Likewise, the probability that three chains of three units triplicate the operations of their central unit is:

$$
v_{1}^{3}=\frac{1}{H K} \sum_{h, h_{1}=1}^{H} \sum_{k, k_{1}=1}^{K}\left(p_{h k_{1}} p_{h_{1} k}\right)^{3}
$$


These terms entail a geometric series of ratio $\sum_{h, h_{1}=1}^{H} \sum_{k, k_{1}=1}^{K}\left(p_{h k_{1}} p_{h_{1} k}\right)$. So if in the organization there are $m$ units that duplicate operations within chains of three units, the sum of this series is:

$$
v_{1}(m)=\frac{1}{H K} \sum_{h, h_{1}=1}^{H} \sum_{k, k_{1}=1}^{K}\left(p_{h k_{1}} p_{h_{1} k}\right)^{2} \frac{1-\left(\sum_{h, h_{1}=1}^{H} \sum_{k, k_{1}=1}^{K} p_{h k_{1}} p_{h_{1} k}\right)^{m}}{1-\sum_{h, h_{1}=1}^{H} \sum_{k, k_{1}=1}^{K} p_{h k_{1}} p_{h_{1} k}}
$$

provided that $\sum_{h, h_{1}=1}^{H} \sum_{k, k_{1}=1}^{K}\left(p_{h k_{1}} p_{h_{1} k}\right) \neq 1$.

If $\sum_{h, h_{1}=1}^{H} \sum_{k, k_{1}=1}^{K}\left(p_{h k_{1}} p_{h_{1} k}\right)<1$ and if $m$ is sufficiently large, the above value can be approximated by its asymptotic limit for $m \rightarrow \infty$ :

$$
v_{1}=\frac{1}{H K} \frac{\sum_{h, h_{1}=1}^{H} \sum_{k, k_{1}=1}^{K}\left(p_{h k_{1}} p_{h_{1} k}\right)^{2}}{1-\sum_{h, h_{1}=1}^{H} \sum_{k, k_{1}=1}^{K} p_{h k_{1}} p_{h_{1} k}}
$$

The above expressions refer to chains of three organizational units where the operations carried out by the central unit are duplicated, triplicated, and so on. We may consider duplication of the operations carried out by a chain of two organizational units, included in a chain of four units. In this case, the stream of information would be $k \rightarrow h_{1} \mapsto k_{1} \rightarrow h_{2} \mapsto k_{2} \rightarrow h$, where $k$ is the information string produced by the first unit, $h_{1}$ and $k_{1}$ are, respectively, the category string and the information string of the second unit, $h_{2}$ and $k_{2}$ are, respectively, the category string and the information string of the third unit, and $h$ is the category string employed by the fourth and last unit.

The probability that two or more chains of four organizational units duplicate operations is:

$$
v_{2}^{2}=\frac{1}{H K} \sum_{h, h_{1}, h_{2}=1}^{H} \sum_{k, k_{1}, k_{2}=1}^{K}\left(p_{h k_{1}} p_{h_{1} k_{2}} p_{h_{2} k}\right)^{2}
$$

where, again, coefficient $1 / H K$ ensures that $0 \leq v_{2}^{2} \leq 1$.

As above, we may consider triplication, quadruplication, and so on. The ensuing series is geometric of ratio $\sum_{h, h_{1}, h_{2}=1}^{H} \sum_{k, k_{1}, k_{2}=1}^{K} p_{h k_{1}} p_{h_{1} k_{2}} p_{h_{2} k}$ and, as above, its sum to the $m$-th order is:

$v_{2}(m)=\frac{1}{H K} \sum_{h, h_{1}, h_{2}=1}^{H} \sum_{k, k_{1}, k_{2}=1}^{K}\left(p_{h k_{1}} p_{h_{1} k_{2}} p_{h_{2} k}\right)^{2} \frac{1-\left(\sum_{h, h_{1}, h_{2}=1}^{H} \sum_{k, k_{1}, k_{2}=1}^{K} p_{h k_{1}} p_{h_{1} k_{2}} p_{h_{2} k}\right)^{m}}{1-\sum_{h, h_{1}, h_{2}=1}^{H} \sum_{k, k_{1}, k_{2}=1}^{K} p_{h k_{1}} p_{h_{1} k_{2}} p_{h_{2} k}}$

provided that $\sum_{h, h_{1}, h_{2}=1}^{H} \sum_{k, k_{1}, k_{2}=1}^{K} p_{h k_{1}} p_{h_{1} k_{2}} p_{h_{2} k} \neq 1$.

As above, the limit of this sum for $m \rightarrow \infty$ is:

$$
v_{2}=\frac{1}{H K} \frac{\sum_{h, h_{1}, h_{2}=1}^{H} \sum_{k, k_{1}, k_{2}=1}^{K}\left(p_{h k_{1}} p_{h_{1} k_{2}} p_{h_{2} k}\right)^{2}}{1-\sum_{h, h_{1}, h_{2}=1}^{H} \sum_{k, k_{1}, k_{2}=1}^{K} p_{h k_{1}} p_{h_{1} k_{2}} p_{h_{2} k}}
$$

provided that $\sum_{h, h_{1}, h_{2}=1}^{H} \sum_{k, k_{1}, k_{2}=1}^{K} p_{h k_{1}} p_{h_{1} k_{2}} p_{h_{2} k}<1$. 
The more units are involved, the less likely it is that duplication of operations occurs. Thus $\left\{v_{1}, v_{2}, \ldots\right\}$ is a decreasing series, whose generic $n$-th term takes the form:

$$
v_{n}=\frac{1}{H K} \frac{\sum_{h, h_{1}, \ldots h_{n}=1}^{H} \sum_{k, k_{1}, \ldots k_{n}=1}^{K}\left(p_{h k_{1}} p_{h_{1} k_{2}} \ldots p_{h_{n-1} k_{n}} p_{h_{n} k}\right)^{2}}{\sum_{h, h_{1}, \ldots h_{n}=1}^{H} \sum_{k, k_{1}, \ldots k_{n}=1}^{K} p_{h k_{1}} p_{h_{1} k_{2}} \ldots p_{h_{n-1} k_{n}} p_{h_{n} k}}
$$

In the end, in a firm with multiple paths up to order $N$ we can measure the extent of operations duplication by means of:

$$
V=\sum_{n=1}^{N} v_{n}
$$

Similarly to equation (3), we can say that if a firm starts with a structure $\mathcal{S}_{*}$ and arrives at a structure $\mathcal{S}^{*}$, the corresponding variation of operations duplication is $\Delta V$ :

$$
\Delta V=\sum_{S_{i}=S_{*}}^{S^{*}} \Delta V_{i}
$$

where $\Delta V_{i}=\left.V\right|_{\mathcal{S}_{i}}-\left.V\right|_{\mathcal{S}_{i-1}}$ and where the series of structures $\left\{\mathcal{S}_{i}\right\}$ extends from $\mathcal{S}_{*}$ to $S^{*}$.

If $\Delta V_{i}<0$ for $\forall i$, then the organization has a spontaneous tendency to move from structure $\mathcal{S}_{*}$ to structure $\mathcal{S}^{*}$.

\section{Applications}

This sections illustrates the meaning of the Lyapunov functions defined above by means of two examples. The first one concerns a stylized comparison between a flexible organization and a hierarchy according to quite a common scheme in the literature. The second one is excerpted from a real case of a firm that, after switching from a classical hierarchy to an extreme form of flexible organization, decreased its level of flexibility at a later stage. In this case, lack of empirical information makes it impossible to apply the equations derived above, but the situation is suggestive of their meaning.

\subsection{Hierarchy vs. Multihierarchy}

A number of comparisons between hierarchies and flexible organizations were prompted by the superior performance of many Japanese firms with respect to their American competitors during the 1980s. Soon, this became a canonical topic in organization science. Western investigators pointed to communication between marketing people and engineers, personnel rotation and flexible teams composed by people from different departments as key factors of the success of Japanese firms. ${ }^{2}$

\footnotetext{
${ }^{2}$ Subsequent investigations yielded a much richer, often different picture of Japanese-style manufacturing. However, here the issue is that of comparing idealized structures that have been widely discussed in the literature.
} 

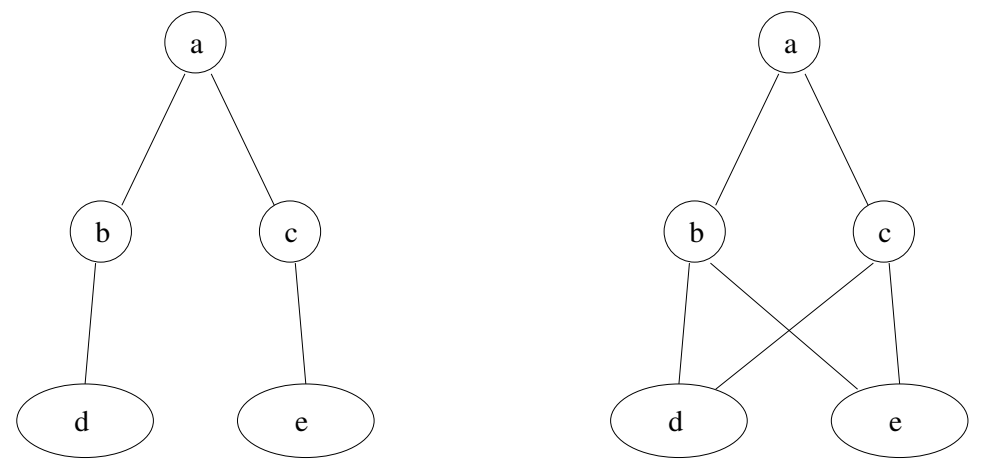

Figure 2: In the "Western" hierarchy on the left, units (b) and (c) rely on information issued by (d) and (e), respectively. In the "Japanese" multihierarchy on the right, units (b) and (c) rely on information issued by both (d) and (e).

Following this interpretation, Japanese-style firms have been characterized as polyarchies, or multihierarchies, i.e. structures where cross-connections between departments originate from superimposition of multiple hierarchies [33] [34] [35] [2]. Figure 2 illustrates the difference between hierarchies and multihierarchies in a highly simplified setting. Both structures entail a commanding unit (a) (the boss) and two functional units (b) and (c) (e.g. engineering and marketing) that process information stemming from two sources (d) and (e) (e.g. technological constraints and market surveys). Contrary to the simple hierarchy, the multihierarchy forces engineers to take account of aesthetical aspects and marketeers to take account of technical aspects.

Let us compute potential functions $U$ and $V$ for the hierarchy and the multihierarchy of figure 2. If a firm is endowed with rational managers and if it operates in a predictable environment, it should move to the structure that has lower $U$ and lower $V$.

Since in the hierarchy each organizational unit receives information from only one other unit and passes on information to only one other unit, each organizational unit has its specific category string and information string, which we may label with numbers following alphabetical ordering. So unit (a) has a category string labelled with $h=1$ and issues an information string labelled with $k=1$, unit (b) has a category string labelled with $h=2$ and issues an information string labelled with $k=2$, and so on.

On the contrary, in the multihierarchy units (b) and (c) must be endowed with similar category strings in order to be able to process both the information strings issued by (d) and the information strings issued by (e). One possibility is that (b) and (c) are endowed with one category each, but one with a large number of \#-characters - i.e. that they become generalists. In this case units (b) and (c) are endowed with the same category strings and produce the same information strings, that are different from those they had in the hierarchy. Another possibility is that (b) and (c) are endowed with two categories each, i.e. that each of them acquires the skills of two specialties. In this case each unit has a different pair of category strings, but may produce the same information string as in the hierarchy.

We shall consider three organizational configurations: the hierarchy, the multihier- 


\begin{tabular}{|c||c|c||c|c||c|c|}
\hline \multicolumn{1}{|c||}{} & \multicolumn{3}{c}{ Hierarchy } & \multicolumn{3}{c}{ Multihierarchy (g) Multihierarchy (s) } \\
unit & $\mathrm{h}$ & $\mathrm{k}$ & $\mathrm{h}$ & $\mathrm{k}$ & $\mathrm{h}$ & $\mathrm{k}$ \\
\hline \hline $\mathrm{a}$ & 1 & 1 & 1 & 1 & 1 & 1 \\
\hline $\mathrm{b}$ & 2 & 2 & $\mathbf{6}$ & $\mathbf{6}$ & $\mathbf{7 , 8}$ & 2 \\
\hline $\mathrm{c}$ & 3 & 3 & $\mathbf{6}$ & $\mathbf{6}$ & $\mathbf{9 , 1 0}$ & 3 \\
\hline $\mathrm{d}$ & 4 & 4 & 4 & 4 & 4 & 4 \\
\hline $\mathrm{e}$ & 5 & 5 & 5 & 5 & 5 & 5 \\
\hline
\end{tabular}

Table 1: The codification of category strings and information strings for the hierarchy, the multihierarchy with generalists $(\mathrm{g})$ and the multihierarchy with multi-skilled specialists (s). Codifications departing from those of the hierarchy have been highlighted.

archy with generalists and the multihierarchy with multi-skilled specialists. Lyapunov functions $U$ and $V$ will be computed in all three cases, and comparisons will be made between the hierarchy and each of the two versions of the multihierarchy.

Table (1) illustrates the codification of category strings and information strings in these three configurations. In the hierarchy, each unit has a category string and issues an information string denoted by a number corresponding to the name of that unit. In the multihierarchy with generalists, units (b) and (c) have the same category string, denoted by $h=6$, and produce the same information string, denoted by $k=6$. For simplicity it is assumed that the category string of unit (a) is still able to capture the information strings issued by (b) and (c). In the multihierarchy with specialists, units (b) and (c) have two category strings each, denoted by $h=7, h=8$ and $h=9, h=10$, respectively. However, they produce the same information strings as in the hierarchy.

Let us compute information waste $U$. The connections between (a) and (b) and between (a) and (c) are characterized by the same amount of information waste in the hierarchy and in the multihierarchy with multi-skilled specialists. So far it regards the multihierarchy with generalists, if we for simplicity assume that $z_{6}=1 / 2\left(z_{2}+z_{3}\right)$, then also in this case these connections contribute the same amount of information waste. Thus, under this assumption the information waste contributed by the connections between (a) and (b) and between (a) and (c) can be ignored.

By applying equation (3) we find that hierarchy, multihierarchy with generalists and multihierarchy with multi-skilled specialists are characterized by the following values of information waste:

$$
\begin{aligned}
U_{H}= & p_{24}\left(z_{4}-s_{2}\right)+p_{35}\left(z_{5}-s_{3}\right) \\
U_{M H g}= & 2 p_{64}\left(z_{4}-s_{6}\right)+2 p_{65}\left(z_{5}-s_{6}\right) \\
U_{M H s}= & p_{74}\left(z_{4}-s_{7}\right)+p_{85}\left(z_{5}-s_{8}\right) \\
& +p_{95}\left(z_{5}-s_{9}\right)+p_{104}\left(z_{4}-s_{10}\right)
\end{aligned}
$$


Let us analyze the meaning of equations (14), (15) and (16). For simplicity, let us assume that $p_{24}=p_{35}=p_{64}=p_{65}=p_{74}=p_{85}=p_{95}=p_{104}$ so probability values can be ignored altogether.

Let us consider the case of a multihierarchy with generalists. It is easy to see that $U_{M H g}<U_{H}$, meaning that a firm has a tendency to switch from the hierarchical to the multihierarchical structure if $s_{6}>1 / 4\left(s_{2}+s_{3}+z_{4}+z_{5}\right)$, i.e. if the category string of the two generalists in (b) and (c) is more specific than the average of the other strings considered. This is nearly impossible because by definition $z_{4} \geq s_{2}$ and $z_{5} \geq s_{3}$ and, furthermore, in order for $h=6$ to be a generalist's category it must be $s_{6} \leq \min \left\{s_{2}, s_{3}\right\}$.

Thus, we must conclude that no spontaneous tendency exists for a firm to switch from a hierarchical structure to a multihierarchical structure implemented by generalists. This does not mean that such a shift may not occur if market unpredictability forces a firm to do so, but that such a shift would take place at the expense of a greater information waste. For instance, if the structure of figure (2) is interpreted in the sense that unit (b) deals with engineering and unit (c) deals with marketing, implementing the multihierarchy by means of generalists means that marketing is so basic that even engineers can do it and that technology is so simple that even the marketing people can understand it, which implies that the firm is unable to exploit the most sophisticated market niches. Although environmental turbulence or other exogenous factors may force a firm to do so, it makes sense that no spontaneous tendency exists for this move.

Let us consider the case of a multihierarchy with multi-skilled specialists. In this case $U_{M H s}<U_{H}$, which means that a firm has a tendency to switch from the hierarchical to the multihierarchical structure if $s_{7}+s_{8}+s_{9}+s_{1} 0>s_{2}+s_{3}+z_{4}+z_{5}$ which, since by definition $z_{4} \geq s_{2}$ and $z_{5} \geq s_{3}$, implies $s_{7}+s_{8}+s_{9}+s_{1} 0>2\left(s_{2}+s_{3}\right)$. Thus, a spontaneous tendency towards a multihierarchy exists only if the category strings of the multihierarchy, though obeying the requirements $s_{7} \leq z_{4}, s_{7} \leq z_{5}, s_{8} \leq z_{4}, s_{8} \leq z_{5}$, $s_{9} \leq z_{4}, s_{9} \leq z_{5}, s_{1} 0 \leq z_{4}, s_{1} 0 \leq z_{5}$, are on average more specific than the category strings of the hierarchy. If the structure of figure (2) is interpreted in the sense that unit (b) deals with engineering and unit (c) deals with marketing, implementing the multihierarchy by means of multi-skilled specialists means that the engineers acquire a profound competence in marketing and that the marketing people acquire a profound competence in engineering. Although theoretically feasible, in practice this is nearly impossible or, at the very least, extremely costly. However, if environmental turbulence or other exogenous factors push a firm towards a multihierarchical structure, enhancing the competences of its organizational units may limit its drawbacks so far it concerns information waste.

Let us consider duplication of operations as expressed by $V$. The hierarchy on the left side of figure 2 does not duplicate any operation. The multihierarchy on the right side of figure 2, if it is implemented with generalists at units (b) and (c), duplicates the operation within the path $z_{4} \rightarrow h_{6} \mapsto k_{6} \rightarrow h_{1}$ as well as the operation within the path $z_{5} \rightarrow h_{6} \mapsto k_{6} \rightarrow h_{1}$. Thus, in this case $V \equiv v_{1}^{2}$. Finally, the multihierarchy on the right side of figure 2 , if it is implemented with multi-skilled specialists, does not duplicate any operation.

In the case of the hierarchy, $H=K=6$. In the case of the multihierarchy with generalists, $H=K=4$. In the case of the multihierarchy with multi-skilled specialists $H=7$ and $K=5$. Equation (12) yields: 


$$
\begin{aligned}
V_{H} & =0 \\
V_{M H g} & =\frac{1}{16}\left(p_{64}^{2} p_{16}^{2}+p_{65}^{2} p_{16}^{2}\right) \\
V_{M H S} & =0
\end{aligned}
$$

When comparing the hierarchy with the multihierarchy with generalists one observes that, since $V_{M H g}>V_{H}$, also from the point of view of operations duplication there exists no endogenous drive to move to a multihierarchy. Eventually, an endogenous drive exists in the opposite direction.

On the contrary, when comparing the hierarchy with the multihierarchy with multiskilled specialists one observes that, since $V_{M H s}=V_{H}$, from the point of view of operations duplication a firm should be indifferent between these two structures. However, the multihierarchy is much more costly and difficult to implement than the hierarchy.

On the whole, we can interpret the empirically observed diffusion of multihierarchical structures as due to forces that are exogenous to the firm, whose negative effects a firm attempts to counterbalance by multi-skilling its employees. To the extent that this is achieved, the drawbacks of a multihierarchy can be (partially) offset and a firm implementing this structure can be economically viable. This theoretical result may shed some light on the emphasis on permanent education and job rotation that characterizes many flexible organizations in the "knowledge-based" economy.

\subsection{A Real Context}

A real context may give an idea of the kind of situations where the above formalism may be applied. The following example is to be meant as a sketchy introduction to possible applications.

Oticon is a Danish producer of hearing aids that, after having been organized as a functional hierarchy until the end of the 1980s, implemented such a radical restructuring to be mentioned as a paramount example of a new emerging structure based on flexibility and teamwork [27] [32]. Oticon's restructuring actually concerned only its headquarters, where R\&D and strategic decisions were made; however, it was far more radical than many re-organizations of research departments, or innovative flexible organizations in research-based firms such as those operating in the bio-tech industry. Thus, the interest of the popular press is definitely justified.

In 1991, Oticon's headquarters moved to a new location where open spaces substituted offices, informal communication substituted paperwork and hierarchy was reduced to two levels [22] [15]. Most importantly, employees were pruned to constitute work teams on whatever project they thought it was worth pursuing - mostly research projects, but there were also projects concerning internal organization as well as marketing projects. The project on which a team decided to work had to be formally 
approved by a committee; however, in practice, the committee approved any project and provided its leader with financial means to "hire" employees in the team. A sort of internal labor market was created. Employees were encouraged to work in several teams at the same time, in some teams as a member, in other teams as the leader. On average, each of the 150 employees worked in 3-4 teams. At any time, about 70 projects were active. Some projects lasted years, others where short-lived.

The reason for such a radical re-organizing was that, during the 1980s, Oticon had lost substantial market shares to competitors who were better able at innovating, notably exploiting digital technologies to build in-the-ear hearing aids. By allowing its researchers to pursue whatever avenue they might conceive, Oticon was able to produce a burst of innovations that quickly restored its market share [31] [37].

However, this extremely flexible structure did not last forever. The fact that any project was allowed to start implied that enormous amounts of resources were wasted, so after some time the CEO had to intervene to stop projects that were clearly unprofitable, a practice that was at odds with the declared management philosophy and which generated obvious discontent [7]. In 1996, Oticon re-organized itself into a structure where, albeit spontaneous formation of work teams was still possible, projects were closely scrutinized before approval, only few of them were actually funded and the internal labor market was abolished. Since 1996 Oticon employees participate on average to $1-2$ different teams instead of $3-4$, which amounts to dropping the number of on-going projects from about 70 to about $30^{3}$.

Whilst the 1991 re-organization was driven by market competition requiring greater innovative capability, the re-organization of 1996 was an internal affair suggested by the need to keep costs under control. Thus, Oticon's story appears as a perfect example of the logic followed in this paper, namely, that environmental unpredictability may push organizations towards flexible structures but nevertheless, to the extent one focuses on endogenous factors, organizations have a natural tendency to move towards more classical configurations. In the light of this framework, Oticon's restructuring of 1991 appears as a correct but excessive response to environmental requirements, which was subsequently curbed by the internal forces operating in the 1996 re-structuring.

The drawbacks of the extremely flexible organization adopted by Oticon from 1991 to 1995 can be easily understood in terms of information waste and duplication of operations. In fact, the empirical literature ascribes the misfunctionings of this period to the following factors [7]:

- Each employee was required to be proficient in several fields. In this way, fruitful interactions with colleagues with quite a different backgrounds would be eased. In the terms of our model, this translates into endowing organizational units with multiple category strings and, possibly, to ascribe them the ability to choose one out of several information strings to produce. However, this was apparently the least serious problem in a high-tech firm such as Oticon, where all employees had a high degree of education.

\footnotetext{
${ }^{3}$ This last figure derives from my own calculations on available data [7]. The ratio of 3.5 (median number of participated teams from 1991 to 1996 ) to 1.5 (median number of participated teams since 1996) is $2 . \overline{3}$. If this ratio reflects into the average number of work teams, they must have passed from 70 in 1991-1996 to 30 since 1996, on average.
} 
- Since work teams competing with one another did not share information, they eventually duplicated research efforts. Evidently, this is an instance of duplication of operations. Furthermore, all work teams engaged in internal politics for approval by the CEO. This is also an instance of operations duplication, in the sense that lobbying activities were duplicated across teams. Apparently, duplication of operations was definitely the most serious problem.

Unfortunately, no empirical study of the Oticon case recorded the birth, death and composition of work teams, the problems that they were called to solve and the solutions that they developed. However, we can speculate how a research could be carried out if data were available.

Each single employee may be considered an organizational unit. Work teams are temporary groupings of these units, wherein some operations are duplicated. For instance, if all teams engage in internal politics in order to influence the CEO, then a "political" sequence of operations is duplicated among all teams. However, sequences of technical operations may be duplicated as well, for instance if several teams start with the same idea (e.g. applying digital electronics to hearing aids), or just if a certain technical process is required by otherwise different projects.

If a careful mapping of Oticon had been recorded, the Lyapunov functions $U$ and $V$ could be computed. The available reports, stressing that duplication of operations was the major problem [7], suggest that in the case of Oticon $V$ may have been more important than $U$. Since this function yields lower values when few operations are duplicated, one may expect $V$ to take a lower value after the 1996 restructuring than in the period 1991-95. Thus, it indicates the direction of endogenous change.

The Oticon example is interesting also because it suggests in what respect a calculation of the extent of information waste and operations duplication might be useful in management. In fact, the interpretation proposed herein suggests that Oticon's 1991 restructuring was made because, although it was clear to everybody that more flexibility was in order, nobody could know how much of it was really needed. In these conditions, the wisest strategy was that of overshooting the target by means of a clearly excessive level of flexibility, which would naturally decrease to its optimal level in subsequent years - the 1995 restructuring. However, overshooting the target is costly, and the more costly the higher managers overshoot. By comparing the extent of information waste and operations duplication in alternative structural arrangements, a manager would be able to assess the extent of flexibility with which her organization would be endowed, and evaluate whether it is worth doing.

\section{Theoretical Framing}

This article presented a technical tool to assess certain structural properties of a firm. Since no tool is neutral with respect to theory, it is in order to conclude by framing it within the wider notion of the theory of the firm, which, as it stands, is split in two streams.

One the one hand, the contractual stream initiated by Coase [6] views the firm as arising because its transactions are more efficient than market transactions. This argu- 
ment has been further deepened by Williamson [40] [41], who ascribed the origin of the inefficiency of market transactions to the possibility opportunistic behavior, which is difficult to detect because of human bounded rationality. March and Simon also wrote in the contractualist stream, though with a wider view [16]. According to March and Simon, the network of contracts that constitutes a firm originates because boundedly rational decision-makers are unable to supervise an environment that is inherently unpredictable, also — but not only — because of opportunistic behavior. By constituting a firm, the actors involved create a place where they enact their own (satisfycing) thumb rules and cognitive maps of causes and effects.

On the other hand, the variably called "resource", "competence", "capability" or "knowledge" -based stream originated by Penrose [26] views a firm as an institution designed to develop and exploit specific competencies. Important developments within this stream included the evolutionary theory of the firm, based on the relative invariance of its routines [23], and the population ecology approach, which builds on routine invariance to apply the mutation-and-selection mechanism to populations of firms [10]. More recently, through the work of Weick and Nooteboom a cognitive stream emerged which understands "resources", "competences", "capabilities" or "knowledge" as deriving from psychic processes [39] [24] [25].

The cognitive trends in both the contractualist and the knowledge-based stream are interesting because they may concur to build a bridge between opposing theories. In fact, the differences between the cognitive version of the contractualist stream (March and Simon) and the cognitive version of the knowledge-based stream (Weick and Nooteboom) are essentially a matter of looking at the firm bottom-up, i.e. starting from the cognitive processes of its members, or top-down, i.e. starting from the collective cognitive processes of the organization as a whole. However different these approaches may be, they are not as inherently irreconcilable as the visions of Coase and Penrose are.

The two measures presented in this article clearly concern menntal processes. In fact, the first one focuses on information categorization, which is the fundamental mechanism of cognition. The second one focuses on the exploration of novel sequences of operations, which is a strategic action of a firm that seeks to cope with an unpredictable environment. Thus, they are deeply embedded within the knowledge-based view.

However, these measures also allow to see the contractualist view as a special case that obtains when no information is wasted and no operations are duplicated. In fact, the mathematical and computational models that have been developed within both the knowledge-based and the contractualist views can be understood within the framework of the Lyapunov functions presented herein.

The contractualist stream produced a series of models where the members of an organization are viewed as information processors. These models succeeded to derive alternative organizational structures from alternative configurations of costs of information processing, costs of information communication and costs of specialization [28] [30] [29] [4] [42] [43] [44]. All of these models can be thought as taking place at the point where no information is wasted and no operations are duplicated, i.e., at the point where the equilibrium described by the Lyapunov functions defined in this article has been achieved. 
The knowledge-based stream produced fewer mathematical and computational models on the interplay between cognition and organizational structures. However, it is interesting to remark that a series of models based on information categorization employes a formalism that is akin to that of the first of our Lyapunov functions [17] [18] [19]. In the case of the knowledge-based stream, its models work at a point that is distant from the equilibrium defined by our Lyapunov functions.

A conciliatory attitude is supported by the empirical evidence on the influence of organizational structure on efficiency, which suggests that although the knowledgebased approach has a larger explanatory power, both approaches are significant [13]. However, one should be aware that the consequences of a theoretical merger based on considering cognitive processes may be much deeper than merely bridging two theoretical streams. In fact, cognition may imply social values, and in this case, contracts may no longer depend solely on individuals' preferences [21].

Finally, it should be remarked that the measures presented in this article imply that managerial action is adaptive to exogeneous uncertainty, rather than proactive. Managers have been assumed to dream of a predictable environment, and to take defensive action if the environment is not so. This is certainly the case in most firms, but there may be exceptions.

\section{A Lyapunov Functions}

A simple way to visualize stable equilibria is to think of a surface in a $n+1$-dimensional space of $n$ state variables, plus one variable for the height of this surface. Henceforth, this surface will be also called a landscape. The $n$-dimensional space of the state variables will be called state space.

The projection of a point of this surface on the $n$-dimensional state space represents a state that a system can attain. Thus, the state of a system may be represented by the position of a ball on the landscape.

According to one possible convention, the lower positions on the surface represent the most preferred states. Thus, a system has a tendency to move from higher positions to lower positions on the surface. Consequently, the bottom of valleys represent stable equilibria. Conversely, the peak of mountains represent unstable equilibria.

According to the opposite convention, the upper positions on the surface represent the most preferred states. Thus, a system has a tendency to move from lower positions to upper positions on the surface. Consequently, the top of mountains represent stable equilibria. Conversely, the bottom of valleys represent unstable equilibria.

Figure 3 illustrates one such surface in a case where $n=2$. The state space is the $X-Y$ plane.

In physics, this landscape is called potential function. Physics makes the convention that the lower positions on this landscape represent the most preferred states. For instance, if this landscape represents the gravitational potential of the earth, this landscape coincides with the commonsense meaning of the word "landscape" with its mountains and valleys and a tendency for a body on top of a mountain to roll down to the bottom of the neighboring valley. Likewise, electrical potential describes the tendency of electrons to move from the negative to the positive pole. 


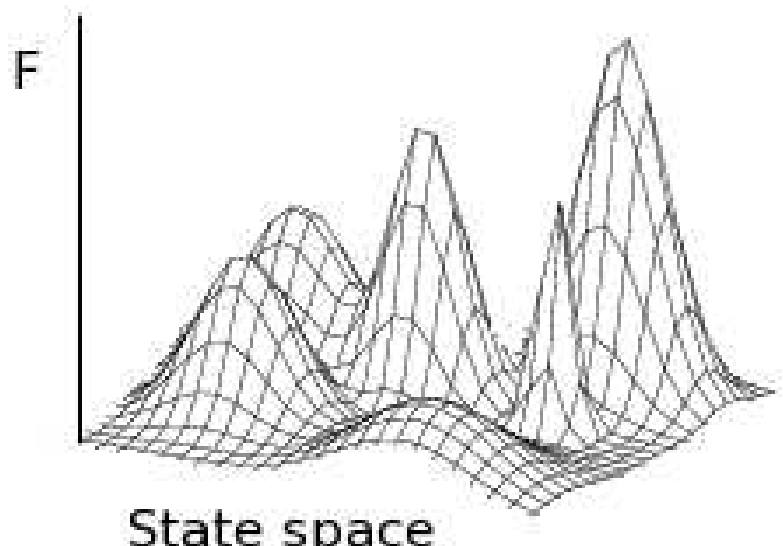

State space

Figure 3: A surface representing equilibria on a two-dimensional state space. According to one convention, the bottom of valleys represent stable equilibria. According to the opposite convention, the top of mountains represent stable equilibria.

In biology this landscape is called fitness function, or fitness landscape. Biology makes the opposite convention to physics: since organisms seek to improve their fitness, the higher points on the surface are the preferred states. So the ball representing a biological system has a tendency to climb mountains, mountain peaks are stable equilibria and the bottom of valleys are unstable equilibria.

Economics makes a similar point when it states that individuals seek to maximize utility: in this case, the landscape is the social welfare function obtained by aggregating individual utility functions. Similarly, firms maximize profits. Also in this case, the effort of maximizing can be represented by climbing a peak.

The conventions employed in physics and respectively in biology and economics are mathematically equivalent to one another. In fact, minimizing a function $F$ is equivalent to maximizing $-F$. Henceforth the convention will be used, that a function $F$ has to be minimized.

The potential functions of physics, the fitness functions of biology and the social welfare functions of economics are Lyapunov functions. The Lyapunov theorem states that, given a system described by state variables $x_{1}, x_{2}, \ldots x_{N}$, the origin of axes is a stable equilibrium if:

1. $\exists F(\mathbf{x}) \in \mathcal{C}^{o}: F(0)=0, F(\mathbf{x})>0$ around the origin;

2. $\frac{\partial F}{\partial x_{1}} d x_{1}+\frac{\partial F}{\partial x_{2}} d x_{2}+\cdots+\frac{\partial F}{\partial x_{N}} d x_{N}<0$.

The Lyapunov theorem can be expressed with respect to the origin of axes without any loss of generality. In fact, any point can be made the origin of the state space by means of a linear transformation.

The Lyapunov theorem says that, if we succeed to find a basin-shaped function such that the state of the system tends to move towards the lowest position, then that 
position is a stable equilibrium. Note that several Lyapunov functions may be defined for a system.

Note also that the Lyapunov theorem provides a sufficient, but not a necessary criterium for stability. Thus, if a Lyapunov function is found, we are certain that the equilibrium is stable. However, if no Lyapunov function is found we cannot conclude that an equilibrium is unstable, and not even that it is not an equilibrium point.

\section{References}

[1] Maurice Allais. La théorie générale des surplus. Économies et Sociétés, 15(1-23):1-718, 1981.

[2] Masahiko Aoki. Horizontal vs. vertical information structure of the firm. American Economic Review, 76(5):971-983, 1986.

[3] W. Ross Ashby. Design for a Brain: The origin of adaptative behavior. Chapman and Hall, London, 1952.

[4] Patrick Bolton and Mathias Dewatripont. The firm as a communication network. The Quarterly Journal of Economics, 109(4):809-839, 1994.

[5] Alfred D. Chandler. Strategy and Structure: Chapters in the history of the industrial enterprise. The MIT Press, Cambridge (MA), 1962.

[6] Ronald H. Coase. The nature of the firm. Economica, 4(16):386-405, 1937.

[7] Nicolai J. Foss. Selective intervention and internal hybrids: Interpreting and learning from the rise and decline of the oticon spaghetti organization. Organization Science, 14(3):331-349, 2003.

[8] Richard M. Goodwin and Lionello F. Punzo. The Dynamics of a Capitalist Economy. Westview Press, Boulder, 1987.

[9] Thomas H. Hammond. Structure, strategy, and the agenda of the firm. In Richard P. Rumelt, Dan E. Schendel, and David J. Teece, editors, Fundamental Issues in Strategy, chapter IV, pages 97-154. Harvard Business School Press, Boston, 1994.

[10] Michael T. Hannan and John Freeman. Organizational Ecology. Harvard University Press, Cambridge (MA), 1988.

[11] John H. Holland. Adaptation in Natural and Artificial Systems. The University of Michigan Press, Ann Arbor, 1975.

[12] John H. Holland. Escaping brittleness: The possibilities of general-purpose learning algorithms applied to parallel rule-based systems. In Ryszard S. Michalski, Jaime G. Carbonell, and Tom M. Mitchell, editors, Machine Learning: An Artificial Intelligence Approach, chapter XX, pages 593-623. Morgan Kaufmann Publishers, Los Altos, 1986. 
[13] Anne Marie Knott and Bill McKelvey. Nirvana efficiency: A comparative test of residual claims and routines. Journal of Economic Behavior \& Organization, 38(4):365-383, 1999.

[14] Paul R. Lawrence and Jay W. Lorsch. Organization and Environment. Harvard University, Graduate School of Business Administration, Boston, 1967.

[15] Bjorn Lovas and Sumantra Ghoshal. Strategy as guided evolution. Strategic Management Journal, 21(9):875-896, 2000.

[16] James G. March and Herbert H. Simon. Organizations. John Wiley \& Sons, New York, 1958.

[17] Luigi Marengo. Coordination and organizational learning in the firm. Journal of Evolutionary Economics, 2(4):313-326, 1992.

[18] Luigi Marengo. Knowledge distribution and coordination in organizations: On some social aspects of the exploitation vs exploration trade-off. Revue Internationale de Systémique, 7(5):553-571, 1993.

[19] Luigi Marengo. Structure, competence and learning in an adaptive model of the firm. In Giovanni Dosi and Franco Malerba, editors, Organization and Strategy in the Evolution of the Entreprise, chapter V, pages 124-154. MacMillan, London, 1996.

[20] Raymond E. Miles and Charles C. Snow. Fit, Failure, and the Hall of Fame. The Free Press, New York, 1994.

[21] Sandro Montresor. Resources, capabilities, competencies and the theory of the firm. Journal of Economic Studies, 31(5):409-434, 2004.

[22] Mette Morsing and Kristian Eiberg, editors. Managing the Unmanageable for a Decade. Oticon A/S, Hellerup, 1998.

[23] Richard R. Nelson and Sidney G. Winter. An Evolutionary Theory of Economic Change. Harvard University Press, Cambridge (MA), 1982.

[24] Bart Nooteboom. Transaction costs, innovation and learning. Technical report, 2004.

[25] Bart Nooteboom. Beyond penrose: A cognitive theory of the firm. Technical Report 34, Tilburg University, 2006.

[26] Edith E.T. Penrose. The Theory of the Growth of the Firm. Basil Blackwell, Oxford, 1959.

[27] Tom Peters. Liberation Management. MacMillan, London, 1992.

[28] Roy Radner. Hierarchy: The economics of managing. Journal of Economic Literature, 30(3):1382-1415, 1992. 
[29] Roy Radner. The organization of decentralized information processing. Econometrica, 61(5):1109-1146, 1993.

[30] Roy Radner and Timothy Van Zandt. Information processing in firms and returns to scale. Annales d'Économie et de Statistique, 7(25-26):265-298, 1992.

[31] Davide Ravasi and Gianmario Verona. Organising the process of knowledge integration: The benefits of structural ambiguity. Scandinavian Journal of Management, 17(1):41-66, 2001.

[32] Suzanne Rivard, Benoit A. Aubert, Michel Patry, Guy Paré, and Heather A. Smith. Information Technology and Organizational Transformation, chapter VII, pages 165-193. Elsevier Butterworth-Heinemann, Amsterdam, 2004.

[33] Raaj K. Sah and Joseph E. Stiglitz. Human fallibility and economic organization. American Economic Review, 75(2):292-297, 1985.

[34] Raaj K. Sah and Joseph E. Stiglitz. The architecture of economic systems: Hierarchies and polyarchies. American Economic Review, 76(4):716-727, 1986.

[35] Raaj K. Sah and Joseph E. Stiglitz. Committees, hierarchies and polyarchies. The Economic Journal, 98(391):451-470, 1988.

[36] James D. Thompson. Organizations in Action: Social science bases of administrative theory. McGraw-Hill, New York, 1967.

[37] Gianmario Verona and Davide Ravasi. Unbundling dynamic capabilities: An exploratory study of continuous product innovation. Industrial and Corporate Change, 12(3):577-606, 2003.

[38] Allen Ward, Jeffrey K. Liker, John J. Cristiano, and Durward K. Sobek II. The second toyota paradox: How delaying decisions can make better cars faster. Sloan Management Review, 36(3):43-61, 1995.

[39] Karl E. Weick. Sensemaking in Organizations. Sage Publications, Thousand Oaks, 1995.

[40] Oliver E. Williamson. Markets and Hierarchies: Analysis and antitrust implications. The Free Press, New York, 1975.

[41] Oliver E. Williamson. The Economic Institutions of Capitalism: Firms, markets, relational contracting. The Free Press, New York, 1985.

[42] Timothy Van Zandt. Hierarchical computation of the resource allocation problem. European Economic Review, 39(3-4):700-708, 1995.

[43] Timothy Van Zandt. Organizations with an endogenous number of information processing agents. In Mukul Majumdar, editor, Organizations with Incomplete Information, chapter VII, pages 239-305. Cambridge University Press, Cambridge, 1998. 
[44] Timothy Van Zandt. Decentralized information processing in the theory of organizations. In Murat Sertel, editor, Contemporary Economic Issues, Vol.4: Economic Behavior and Design, chapter VII, pages 125-160. MacMillan, London, 1999. 